\title{
CHINA AND JAPAN
}





\title{
CHINA AND JAPAN

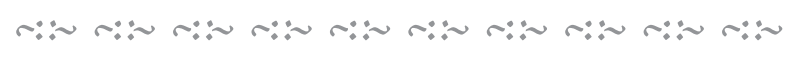 Facing History
}

\section{EZRA F. VOGEL}

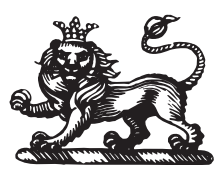 \\ The Belknap Press of Harvard University Press \\ CAMBRIDGE, MASSACHUSETTS \\ LONDON, ENGLAND \\ 2019
}




\section{Copyright (C) 2019 by Ezra F. Vogel}

All rights reserved

Printed in the United States of America

First printing

Design by Dean Bornstein

Jacket photographs: (top) Door knockers at Longhua Ta (Longhua Temple and Pagoda), Shanghai, China/Pictures from History/David Henley/Bridgeman Images; (bottom) Detail of door, Kyoto, Japan/ Jim Holmes/Design Pics/Bridgeman Images

$$
\begin{gathered}
9780674240766 \text { (EPUB) } \\
9780674240780 \text { (MOBI) } \\
9780674240759 \text { (PDF) }
\end{gathered}
$$

The Library of Congress has cataloged the printed edition as follows:

Names: Vogel, Ezra F., author.

Title: China and Japan : facing history / Ezra F. Vogel.

Description: Cambridge, Massachusetts : The Belknap Press of Harvard University

Press, 2019. | Includes bibliographical references and index.

Identifiers: LCCN 20I8053335 | ISBN 9780674916579 (alk. paper)

Subjects: LCsH: China-Relations-Japan. | Japan-Relations-China. |

China_Foreign relations-Japan. | Japan-Foreign relations-China. |

China-Civilization-Japanese influences. | Japan-Civilization-

Chinese influences.

Classification: LCC DS740.5.J3 V59 2019 | DDC 327.51052-dc23

LC record available at https://lccn.loc.gov/2018053335 